\title{
Theoretical investigation of thermal effects in an adiabatic chromatographic column using a lumped kinetic model incorporating heat transfer resistances
}

\author{
Shamsul Qamar, ${ }^{,+\dagger, \ddagger}$ Nadia Kiran, ${ }^{\ddagger}$ Talha Anwar, ${ }^{\ddagger}$ Sameena Bibi, ${ }^{\Uparrow}$ and Andreas \\ Seidel-Morgenstern ${ }^{\dagger}$ \\ $\dagger^{*}$ Max Planck Institute for Dynamics of Complex Technical Systems, Magdeburg, Germany \\ $\ddagger$ COMSATS Institute of Information Technology, Islamabad, Pakistan \\ \Air University, PAF Complex, Sector E-9 Islamabad, Pakistan \\ E-mail: *qamar@mpi-magdeburg.mpg.de
}

\begin{abstract}
A non-equilibrium non-isothermal lumped kinetic model (LKM) is analytically and numerically investigated to evaluate the effects of inherent temperature fluctuations in an adiabatic chromatographic column. The model comprises of convectiondiffusion partial differential equations quantifying mass and energy balances in the mobile phase coupled with differential and algebraic equations for mass and energy in the stationary phase. Besides two mass transfer coefficients, two heat transfer coefficients are involved in the model equations. The properties of the considered model are investigated for linear concentration and temperature dependencies of the equilibrium loadings. The Laplace transformation and eigen-decomposition techniques are utilized to solve the set of equations. These solutions are helpful for understanding,
\end{abstract}


analyzing and interpreting the propagation speeds and shapes of both concentration and thermal fronts migrating in chromatographic columns. The moment generating property of the Laplace domain solutions is employed to obtain explicit analytical temporal moments of the concentration and energy profiles which provide instructive tools to analyze the properties of the model considered and to estimate unknown model parameters from measured transients. For illustration several case studies are carried out assuming realistic model parameters. The applicability range of the analytical solutions derived is assessed by comparing selected specific results with numerical results of a non-equilibrium and non-isothermal model considering nonlinear adsorption isotherms.

\section{Introduction:}

Liquid chromatography is one of the most effective and flexible separation techniques. The concept is successfully applied to perform various complex separation processes with analytical or preparative purpose. ${ }^{1-5}$ The separation of sample components is due to specific distributions of the components to be separated between the two phases involved. The solid phase remains fixed in the column, while the liquid phase is mobile and carries mixtures of dissolved components through the column. Due to different migration speeds of the solutes, the separated peaks can be withdrawn as different fractions at the end of the column with the desired purity. Since binding of the solute by adsorption is an exothermic and desorption is endothermic, there is a dynamic heat exchange between the two phases. These thermal effects are usually neglected in describing liquid chromatography by a) considering heat capacities of the two phases large as compared to the adsorption enthalpies and b) assuming a sufficiently large value of thermal conductivity in order to maintain a uniform temperature in the column throughout the process. Thus, most process mod-

els of adsorption chromatography assume isothermal conditions in liquid chromatography. 
Cerro and Smith ${ }^{6}$ analyzed the larger thermal effects in the gas chromatography. Later on Haynes ${ }^{7}$ studied that how the shape of the distribution equilibria is connected to heat effects. This work was based on evaluating temporal moments which inspired Zhong and Meunier ${ }^{8}$ to study interferences in non-isothermal and non-equilibrium perturbations chromatography. They determined analytical expressions for the first and second temporal moments. Further comprehensive studies devoted to evaluate heat effects in gas chromatography using solid non-catalytic and catalytic packings are available. ${ }^{9-12}$ In the case of using liquid mobile phase, there are more contributions in which thermal effects were studied. ${ }^{13-24}$

Several mass transfer based models have been established and applied to quantify liquid chromatography assuming constant flow rates. Among those chromatographic models, which differ in the number of model parameters, the most frequently used models include the ideal model, the linear driving force model, the equilibrium dispersive model (EDM), the lumped kinetic model (LKM), and the general rate model (GRM). ${ }^{3-5}$ All these models require an input information regarding the thermodynamic equilibrium for the distribution of components between the mobile and stationary phases. Each model has different limitations and level of complexity for the process description. This work focusing on the analytical and numerical approximation of a non-isothermal and non-equilibrium LKM. The LKM incorporates the rate of variation of the local concentration of solute in solid phase and local deviation from equilibrium concentrations and exploits two kinetic parameters. The simpler EDM assumes that the mass transfer between fluid and solid is of infinite rate. It requires just one parameter. The more complex GRM accounts for axial dispersion and several mass transfer resistances. The considered non-equilibrium LKM is a good compromise: it is formed by convection-diffusion partial differential balance equations in the mobile phase coupled with differential equations for masses in the stationary phase along with equilibrium equations for isotherms. 
The aim of this contribution is to analyze with the LKM the magnitude and consequences of temperature fluctuations in a liquid chromatographic column. The joint occurrence of mass and thermal fronts is demonstrated. Parameters that significantly affect the temperature gradients are identified. The current research work extends and generalizes our previous analysis for the simpler linearized non-isothermal EDM. ${ }^{24}$ In contrast to the non-isothermal EDM, the more realistic and more flexible non-isothermal LKM incorporates both mass and heat transfer resistances in each of the solid phase balance equations. Assuming a simple linearized equilibrium function, the Laplace transformation and eigen-decomposition technique are utilized to derive analytical expressions for concentration and energy profiles in the Laplace domain. ${ }^{25-27}$ A numerical Laplace inversion technique is applied for back transformation of the solutions in the actual time domain. ${ }^{28}$ Moreover, analytical expressions of temporal moments are derived from the Laplace domain solutions using the moment generating property of the Laplace transformation. For efficient parameter estimation, the usefulness and relevance of matching theoretically and experimentally determined moments is well-known. Detailed studies of moment analysis are available. ${ }^{5,24,27,29-41}$ In order to validate analytical results obtained for linearized isotherms, a high resolution finite volume scheme (HR-FVS) is utilized to generate accurate numerical results of the same model equations for nonlinear isotherm. ${ }^{20,42} \mathrm{~A}$ detailed analysis of the considered non-equilibrium and non-isothermal LKM model is provided through several case studies. In summary, this theoretical analysis discusses several aspects regarding non-isothermal and non-equilibrium LKM that have not been yet taken into account in this detail. Contrary to the simpler non-isothermal EDM that assumes both mass and heat transfer rates as infinite,${ }^{24}$ the current model equations consider both mass and heat transfer resistances exploiting four kinetic parameters. The analytical solutions of governing equations are derived through the Laplace transformation and eigen-decomposition techniques. Different influences of the four kinetic and thermodynamic parameters on the concentration and 
temperature profiles are comprehensively analyzed and addressed. For further analysis of the process, expressions for the first two moments for concentration and temperature are derived. Eventually, the considered HR-FVS is applied to numerically approximate the model equation also for nonlinear isotherms. This allows to compare and evaluate the assumption of linear isotherm. ${ }^{20,42}$

It should be mentioned here briefly that friction between the flowing eluent and the liquid chromatographic column generates also viscous heat. As a result, the temperature along the column can significantly rise. ${ }^{43}$ Furthermore, non-adiabatic conditions under radial temperature gradients can be generated that may seriously reduce column efficiency. Thus, precautions might be useful to minimize losses of heat through wall of the column. Further details about these phenomena, not considered in this manuscript, can be found in $^{43}$ and references therein.

The contents of this paper are arranged as follows. In Section 2, the studied non-isothermal LKM is briefly introduced. Section 3 refers the reader to Appendices S1 and S2 for the derivation of analytical solutions and moments. In Section 4, several case studies of practical importance are provided. Finally, conclusions are given in Section 5.

\section{The non-isothermal lumped kinetic model}

A chromatographic column packed with uniform spherical porous particles is considered, through which a single-solute flows along with an inert carrier. A rectangular pulse of concentration is then injected to the column which is initially at equilibrium state and not necessarily clean. The following simplifying assumptions are considered: ${ }^{8}$ (i) The mobile phase is incompressible. (ii) No radial concentration gradients exist in the column. (iii) No interaction takes place between the carrier and the solid phase. (iv) The equilibrium relationships are linearized. Generally, adsorption equilibrium relationships are 
nonlinear, however, the current linear assumptions are valid for small changes in the concentration and temperature. (v) A change in temperature does not effects the physical properties like density, viscosity and heat capacity, and transport coefficients like axialdispersion and axial-heat-conductivity. (vi) The axial-dispersion coefficient and the axialheat-conductivity coefficient are not depending on the flow rate. (vii) The overall rate of solute adsorption is represented by the linear driving force (LDF) model. (viii) The heat transfer resistance in the particle is concentrated at the surface of the particle.

In the light of above assumptions, the one-dimensional mass balance of the single-component LKM in the mobile phase is given as ${ }^{5,8}$

$$
\frac{\partial c}{\partial t}+u \frac{\partial c}{\partial z}=D_{L} \frac{\partial^{2} c}{\partial z^{2}}-F \frac{\partial q}{\partial t}
$$

The corresponding heat balance, additionally considered in this paper, is expressed as ${ }^{5,8}$

$$
\frac{\partial T}{\partial t}+u \frac{\partial T}{\partial z}=\frac{\lambda_{L}}{C_{L}} \frac{\partial^{2} T}{\partial z^{2}}+F \frac{\left(-\Delta H_{A}\right)}{C_{L}} \frac{\partial q}{\partial t}-F \frac{C_{S}}{C_{L}} \frac{\partial T_{S}}{\partial t}
$$

where

$$
C_{S}=\rho^{S} c_{p}^{S}, \quad C_{L}=\rho^{L} c_{p}^{L}, \quad F=\frac{1-\epsilon}{\epsilon}
$$

In the above equations, $c$ represents the concentration of solute in the mobile phase, $T$ stands for temperature of the mobile phase, $T_{S}$ represents temperature in the solid phase, $q$ is the non-equilibrium mean loading of solute in the solid phase, $u$ is the interstitial velocity, $\Delta H_{A}$ is the enthalpy of adsorption, $D_{L}$ denotes the axial dispersion coefficient, $\lambda_{L}$ is the heat conductivity coefficient, $z$ is the axial coordinate, and $t$ is the time coordinate. Moreover, in Eq. (3), $\rho^{S}$ denotes the density per unit volume in the solid phase, $\epsilon$ represents the external porosity, $c_{p}^{S}$ represents the heat capacity in the solid phase, $\rho^{L}$ denotes the 
density per unit volume in the mobile phase, $c_{p}^{L}$ represents the heat capacity in the mobile phase, and the subscripts $S$ and $L$ stands for solid and liquid phase, respectively.

The well-known mass balance and the newly introduced heat balance for the solid phase are given as $^{5,8}$

$$
\begin{aligned}
\frac{\partial q}{\partial t} & =k\left(q^{*}-q\right) \\
\frac{\partial T_{S}}{\partial t} & =\frac{\left(-\Delta H_{\mathrm{A}}\right)}{C_{S}} \frac{\partial q}{\partial t}+\frac{3 h}{R_{p} C_{S}}\left(T-T_{S}\right) .
\end{aligned}
$$

In above equations $k$ denotes a constant apparent mass transfer rate coefficient, $h$ is the heat transfer coefficient between liquid phase and adsorbent solid phase, $q^{*}$ is the equilibrium concentration in the solid phase and $R_{p}$ represents radius of the particle. Sajonz et al. ${ }^{44}$ have extended the shock layer thickness theory to systems having concentration-dependent mass transfer rate coefficients and compared their calculated values with experimental data. While, Kaczmarski et al. ${ }^{45}$ made reevaluation of previous experimental data on the mass transfer kinetics of bovine serum albumin (BSA) in anion-exchange chromatography under nonlinear conditions using the general rate model and the pore diffusion model of chromatography. In both articles, the authors observed that the apparent rate coefficient increases with increasing concentration. This aspect is ignored below.

The current non-isothermal LKM in Eqs. (1)-(5) reduces to the non-isothermal EDM of our previous article ${ }^{24}$ for large mass and heat transfer coefficents, i.e. for $k \rightarrow \infty$ and $h \rightarrow \infty$. The temperature influences the amount of solute adsorbed which is expressed by following phase equilibrium relation ${ }^{24}$

$$
q^{*}\left(c, T_{S}\right)=a_{\mathrm{ref}} e^{\left(\frac{\left(-\Delta H_{\mathrm{A}}\right)}{R_{g}}\left(\frac{1}{T_{S}}-\frac{1}{T_{\mathrm{ref}}}\right)\right)} c .
$$

In the above equation, $a_{\text {ref }}$ denotes the Henry's constant at reference $T_{\text {ref }}$ and $R_{g}$ stands 
for the general gas constant.

Let us re-define the liquid and solid phase concentrations as

$$
c_{1}=c, \quad q_{1}=q
$$

and introduce the following transformations for temperatures in the liquid and solid phases:

$$
c_{2}=T-T_{\text {ref }}, \quad q_{2}=T_{\mathrm{S}}-T_{\text {ref }} .
$$

Similarly

$$
q_{1}^{*}=q^{*}\left(c, T_{S}\right) .
$$

In the above definitions, the subscript 1 is used for concentrations and the subscript 2 is used for temperatures. Such new variables are very helpful to write the model equations in a unified manner that facilitates the application of our proposed analytical solution technique. After putting Eq. (5) in Eq. (2) and using Eqs. (7) and (8) in Eqs. (1)-(4), we get

$$
\begin{aligned}
\frac{\partial c_{1}}{\partial t}+u \frac{\partial c_{1}}{\partial z} & =D_{L} \frac{\partial^{2} c_{1}}{\partial z^{2}}-F \frac{\partial q_{1}}{\partial t} \\
\frac{\partial c_{2}}{\partial t}+u \frac{\partial c_{2}}{\partial z} & =\frac{\lambda_{L}}{C_{L}} \frac{\partial^{2} c_{2}}{\partial z^{2}}+F \frac{\left(-\Delta H_{A}\right)}{C_{L}} \frac{\partial q_{1}}{\partial t}-F \frac{C_{S}}{C_{L}} \frac{\partial q_{2}}{\partial t} \\
\frac{\partial q_{1}}{\partial t} & =k\left(q_{1}^{*}-q_{1}\right), \\
\frac{\partial q_{2}}{\partial t} & =\frac{\left(-\Delta H_{A}\right)}{C_{S}} \frac{\partial q_{1}}{\partial t}+\frac{3 h}{R_{p} C_{S}}\left(c_{2}-q_{2}\right) .
\end{aligned}
$$

In order to reduce the number of parameters involved in the model equations and to 
simplify our notations, the following dimensionless quantities are considered:

$$
\begin{aligned}
x & =\frac{z}{L}, \quad \tau=t \frac{u}{L}, \\
P e_{c} & =\frac{u / L}{D_{L} / L^{2}}, \quad P e_{T}=\frac{u / L}{\lambda_{L} /\left(C_{L} L^{2}\right)}, \quad B i=\frac{k}{u / L}, \\
H_{L} & =\frac{3 h}{(u / L) R_{p} C_{L}}, \quad H_{S}=\frac{3 h}{(u / L) R_{p} C_{S}} .
\end{aligned}
$$

Here, $L$ denotes the column length and $B i$ is the Biot number comparing convection and mass transfer. Moreover, $P e_{c}$ and $P e_{T}$ are the Peclet numbers for mass and heat, respectively.

On introducing Eqs. (14)-(16) in Eqs. (10)-(13), we get

$$
\begin{aligned}
& \frac{\partial c_{1}}{\partial \tau}+\frac{\partial c_{1}}{\partial x}=\frac{1}{P e_{c}} \frac{\partial^{2} c_{1}}{\partial x^{2}}-F \frac{\partial q_{1}}{\partial \tau} \\
& \frac{\partial c_{2}}{\partial \tau}+\frac{\partial c_{2}}{\partial x}-\frac{1}{P e_{h}} \frac{\partial^{2} c_{2}}{\partial x^{2}}=-F H_{L}\left(c_{2}-q_{2}\right) \\
& \frac{\partial q_{1}}{\partial \tau}=B i\left(q_{1}^{*}-q_{1}\right) \\
& \frac{\partial q_{2}}{\partial \tau}=\frac{\left(-\Delta H_{A}\right)}{C_{S}} \frac{\partial q_{1}}{\partial \tau}+H_{S}\left(c_{2}-q_{2}\right) .
\end{aligned}
$$

For small changes in the concentration and temperature profiles, the linear Taylor expansion can be used to linearize the equilibrium relation between the solid and liquid phases in Eq. (6). It is given as ${ }^{24}$

$$
q_{1}^{*}\left(c, T_{S}\right) \approx q_{1}^{*}\left(c_{\mathrm{ref}}, T_{\mathrm{ref}}\right)+\left.\frac{\partial q_{1}^{*}}{\partial T_{S}}\right|_{\left(c_{\mathrm{ref}}, T_{\mathrm{ref}}\right)}\left(T_{S}-T_{\mathrm{ref}}\right)+\left.\frac{\partial q_{1}^{*}}{\partial c}\right|_{\left(c_{\mathrm{ref}}, T_{\mathrm{ref}}\right)}\left(c-c_{\mathrm{ref}}\right) .
$$

After this simplification, the above expression takes the following general form: ${ }^{24}$

$$
q_{1}^{*}\left(c_{1}, q_{2}\right)=R_{1} c_{1}+R_{2} q_{2}
$$


where

$$
R_{1}=a_{\mathrm{ref}}, \quad R_{2}=-\frac{\left(-\Delta H_{A}\right) a_{\mathrm{ref}} c_{\mathrm{ref}}}{R_{g} T_{\mathrm{ref}}^{2}}
$$

For an initially equilibrated column, the initial conditions are given as

$$
c_{1}(0, x)=c_{1, \text { init }}, \quad q_{1}(0, x)=q_{1, \text { init }}^{*}, \quad c_{2}(0, x)=0, \quad q_{2}(0, x)=0
$$

Here, $c_{1, \text { init }}$ and $q_{1 \text {,init }}^{*}$ represent the initial equilibrium concentrations in the liquid and solid phases, respectively. Moreover, it is assumed that column is at reference temperature initially. The following boundary conditions (BCs) are considered at both ends of the column $^{24}$

$$
\begin{aligned}
& c_{1}(\tau, 0)=\left\{\begin{array}{lc}
c_{1, \text { inj }}, & \text { if } 0 \leq \tau \leq \tau_{\text {inj }} \\
0, & \tau>\tau_{\text {inj }}
\end{array} \quad, \quad \frac{\partial c_{1}}{\partial x}(\tau, \infty)=0,\right.
\end{aligned}
$$

where

$$
c_{2, \mathrm{inj}}=T_{\mathrm{inj}}-T_{\text {ref }}, \quad \tau_{\mathrm{inj}}=\frac{u t_{\mathrm{inj}}}{L} .
$$

Here, $c_{1, \text { inj }}$ and $T_{\text {inj }}$ denote the concentration and temperature of the rectangular pulse which is injected to the column up to the time $t_{\text {inj. }}$. The Peclet numbers of concentration and temperature are generally high in liquid chromatography and, thus, justifying the use of Dirichlet boundary conditions. 


\section{Analytical solutions and moments}

Analytical solutions and temporal moments of the above model equations for the considered initial and boundary conditions (c.f. Eqs. (17)-(26)) are presented in Appendices S1 and S2, respectively. The Laplace transformation, eigen-decomposition technique and moment generating property of the Laplace domain solutions are used as basic tools to generate the analytical solutions and moments. ${ }^{24,26}$ The numerical Laplace inversion technique of Durbin $^{28}$ is applied to back transform Laplace domain solutions in the actual time domain.

\section{Numerical case studies}

In this section, we provide various case studies to analyze the outlet profiles of liquid phase concentration $\left(c_{1}\right)$ and temperature $\left(c_{2}\right)$ obtained from the semi-analytical solutions (c.f. Eqs. (S1-30), (S1-31) and (S1-34)) of the linearized non-isothermal LKM based on the isotherm given by Eq. (22). A second order accurate finite volume scheme (FVS) is in addition also used to numerically solve the differential forms of nonlinear model equations (c.f. Eqs. (17)-(17)) based on the nonlinear isotherm given by Eq. (6). A comparison of analytical and numerical results is provided to evaluate the applicability range of our analytical solutions. In all figures, just the liquid phase concentration and temperature profiles at the column outlet (at $x=1$ ) are plotted. The reference parameters used in the test problems are listed in Table 1. These model parameters are chosen in accordance with ranges typically encountered in liquid chromatography applications. 


\section{A comparison of isothermal $\left(\Delta H_{A}=0\right)$ and non-isothermal $\left(\Delta H_{A} \neq\right.$ $0)$ cases.}

Figure 1 compares the results of both EDM and LKM for isothermal $\left(\Delta H_{A}=0\right)$ and non-isothermal $\left(\Delta H_{A} \neq 0\right)$ cases. The initial and inlet temperatures were set equal to the reference temperature $\left(T_{\text {init }}=T_{\text {inj }}=T_{\text {ref }}\right)$. Figure $1(\mathrm{a})$ shows that in the non-isothermal case temperature fluctuations have no noticeable effect on the concentration profile $c_{1}$. The profiles of LKM coincide with that of EDM for relatively large mass and heat transfer coefficients, i.e. for $k=200 \mathrm{~min}^{-1}$ and $h=10 \mathrm{~J} / \mathrm{min} \mathrm{cm}^{2} \mathrm{~K}$. On the other hand, they considerably deviate from each other for smaller mass and heat transfer coefficients, such as for $k=1 \mathrm{~min}^{-1}$ and $h=0.1 \mathrm{~J} / \mathrm{min} \mathrm{cm}^{2} K$. Figure 1(b) emphasizes that for zero enthalpy of adsorption, i.e. for isothermal case, the temperature profile $c_{2}$ remains constant. On the other hand, a moderate value of the enthalpy of adsorption (i.e. $\Delta H_{A}=-2 \mathrm{~kJ} / \mathrm{mol}$ )

already affects $c_{2}$ visibly. It can be noticed that for this case adsorption rises the temperature at the peak front to about $2.5 \mathrm{~K}$, while desorption clearly reduces the temperature at the peak tail below the reference value. After elution, the temperature attains again its reference value. Profiles predicted by LKM are wider due to larger mass and heat transfer resistance. It can be further noticed that both concentration and temperature fronts are traveling at different different speeds. For the case considered their mean retention times (c.f. Eq. (S2-5)) are given as $\mu_{11}=14.2 \mathrm{~min}$ and $\mu_{22}=12.92 \mathrm{~min}$, respectively.

\section{Effects of mass and heat transfer coefficients.}

Figures $2(\mathrm{a}) \&(\mathrm{~b})$ show in more detail the influence of the mass transfer coefficient $k$ on the concentration and temperature profiles for a fixed heat transfer coefficient $h=$ $10 \mathrm{~J} / \mathrm{min} \mathrm{cm}^{2} \mathrm{~K}$. In contrast, Figures $2(\mathrm{c}) \&(\mathrm{~d})$ display the effect of $h$ on the profiles for $k=200 \mathrm{~min}^{-1}$. Here, we have taken $\Delta H_{A}=-2 \mathrm{~kJ} / \mathrm{mol}$ and again $T_{\text {init }}=T_{\text {inj }}=T_{\text {ref }}$. It 
can be observed that the heights of both concentration and temperature profiles increase and the peaks become narrower on increasing the value of $k$ from $1 \mathrm{~min}^{-1}$ to $200 \mathrm{~min}^{-1}$. On the other hand, $h$ has in the range considered negligible influence on the concentration profile but significantly effects the width of the temperature profile. Once again, the profiles of EDM and LKM coincide for large mass and heat transfer coefficients.

\section{Influence of model parameters on the temperature fluctuations.}

Figure 3 shows the effects of mass transfer coefficient $k$, the heat transfer coefficient $h$, the enthalpy of adsorption $\Delta H_{A}$ and ratio of specific heats $C_{S} / C_{L}$ on the temperature differences between liquid and solid phases, i.e. on $\left(c_{2}-q_{2}=T-T_{S}\right)$. It can be observed that how difference between the liquid and solid phases temperatures increases on reducing the values of mass and heat transfer coefficients. The amplitude of temperature fluctuations of course also cleanly increases on increasing the $\left|\Delta H_{A}\right|$. Moreover, the amplitude of temperature fluctuations is larger for the ratio $C_{S} / C_{L}=1$. For this value of the heat capacity ratio, the concentration and temperature profiles are traveling at closer speeds

inside the column. Because of the considered relative low enthalpy of adsorption, the resulting temperature fluctuations have in the cases considered no visible effect on the concentration profiles and are, therefore, not displayed here.

\section{Effect of adsorption enthalpy $\Delta H_{A}$ for $T_{\text {inj }}=T_{\text {init }}$ and $C_{S} / C_{L}=1$.}

Figure 4 displays elution profiles at different values of the enthalpy of adsorption $\Delta H_{A}$. One can easily notice that analytical results for the linearized isotherm (c.f. Eq. (22)) and numerical results of the suggested HR-FVS for the nonlinear isotherm (c.f. Eq. (6)) start moving away from each other when the enthalpy of adsorption, $\left|\Delta H_{A}\right|$, is raised from $2 \mathrm{~kJ} / \mathrm{mol}$ to $10 \mathrm{~kJ} / \mathrm{mol}$. The large value of enthalpy of adsorption is a major source for 
large temperature fluctuations. These predictions clearly support but also restrict the assumptions regarding the linearization of the isotherm for moderate values of the enthalpies of adsorption. For $\Delta H_{A}=-10 \mathrm{~kJ} / \mathrm{mol}$ the predicted temperature deviations already

exceed $1 K$ (Figure 4(b)). Thereby, our analytical solution over predicts the temperature furcations from isothermal condition in comparison to the more realistic numerical solution and predicts sharper peaks.

\section{Influence of "non-matching" temperature of injection $\left(T_{\mathrm{inj}} \neq T_{\text {init }}\right)$}

Figure 5 shows the behaviors liquid temperature $c_{2}$ and solid temperature $q_{2}$ on varying the injection temperature. In Figure 5(a), it can be clearly observed that height of fast moving adsorption peak of the temperature increases in the case of a hot injection $\left(T_{\mathrm{inj}}>T_{\mathrm{init}}\right)$. The later occurring temperature drops caused by endothermic desorption gradually shrink when the temperatures of injection are increased ( $300 K$ vs. $310 K)$. Contrary to the previous case, as can be seen in Figure 5(b), the temperature desorption peak enlarges in the negative downward direction for the cold injection case $\left(T_{\mathrm{inj}}<T_{\mathrm{init}}\right)$. In such a case the exothermal adsorption peak diminishes. However, in both case considered, because of the considered low enthalpy of adsorption, the depicted small temperature fluctuations have no visible effect on the concentration profiles and are, thus, not displayed here.

\section{Effect of adsorption equilibrium constant $a_{\text {ref }}$.}

Figures 6 displays the influence of reference Henry's constant $a_{\text {ref }}$ on the concentration and temperature profiles. Again the reference parameters and $\Delta H_{A}=-2 \mathrm{~kJ} / \mathrm{mol}$ are used. The well-known effect can be noticed, that is on increasing $a_{\text {ref }}$, profiles shift towards larger retention times and their band broadening increases. At $a_{\text {ref }}=1.2$ both concentration and temperature peaks are propagating at almost same speed. For the parameters considered, 
in the case of larger $a_{\mathrm{ref}}>1.2$, the concentration profiles and the slow desorption peaks of the temperature profile are coupled, while the positive temperature perturbation caused by adsorption is decoupled and travels ahead in all cases.

\section{Effect of the ratio $C_{S} / C_{L}$ and $T_{\text {init }} \neq T_{\text {inj }}$}

Figure 7 demonstrates the effects of varying the ratio $C_{S} / C_{L}$, which is assumed equal to one in the above calculations, and the injection temperature $T_{\mathrm{inj}}$ on the concentration and temperature profiles. This heat capacity ratio influences the retention times of concentration and temperature fronts. Therefore, it is elaborated in more detail.

In Figures 7(a)\&(b), we have considered $C_{S}=40 \mathrm{~J} / \mathrm{cm}^{3} \mathrm{~K}, C_{L}=4 \mathrm{~J} / \mathrm{cm}^{3} \mathrm{~K}$ and different injection temperatures. For this $C_{S} / C_{L}=10$ ratio, the concentration profile is moving faster than the thermal wave. Therefore, the fast moving adsorption, related to positive peak of thermal wave, is coupled with the speedy concentration profile. On the other hand, the slow decoupled negative desorption peak of the temperature exits the column later on. For the considered test case, the values of mean retention times are $\mu_{11}=14.17 \mathrm{~min}$ and $\mu_{22}=69.17 \mathrm{~min}$. Herby, $\mu_{11}$ illustrates the mean retention time of the coupled mass and energy adsorption peaks. Whereas, $\mu_{22}$ represents the mean retention time of the decoupled temperature peak related to the desorption (see Figure 7(a)). Furthermore, $\sigma_{11}=4.04 \mathrm{~min}^{2}$ describes the variance of the coupled concentration and temperature adsorption peaks, while, $\sigma_{22}=13.49 \mathrm{~min}^{2}$ denotes the variance of the decoupled energy desorption peak. It can be further observed that injection temperature effects the slow moving desorption peak, while the fast moving adsorption peak stays unaffected. Figures $7(\mathrm{c}) \&(\mathrm{~d})$ provide the corresponding plots of the profiles for $C_{S}=4 \mathrm{~J} / \mathrm{cm}^{3} \mathrm{~K}$ and $C_{L}=40 \mathrm{~J} / \mathrm{cm}^{3} \mathrm{~K}$. In this hypothetical case, the velocity of the concentration profile is slower than the thermal wave. Thus, the negative desorption peak of the temperature is coupled with the concentration profile. In this case, $\mu_{11}=14.17$ min represents the reten- 
tion time of the slowly moving coupled concentration and temperature desorption peaks. While, $\mu_{22}=7.29 \mathrm{~min}$ represents the retention time of fast temperature adsorption peak (see Figures 7(c)\&(d)). Moreover, the variance of the coupled slow peaks of concentration and temperature is $\sigma_{11}=3.59 \mathrm{~min}^{2}$, while the variance of the faster energy adsorption peak is just $\sigma_{22}=3.23 \mathrm{~min}^{2}$. It can be further seen that injection temperature effects the fast moving adsorption peak, while the slow moving desorption peak stays unaffected. Thus, the decoupled thermal waves have been influenced by the injection temperatures in both cases.

\section{Effects of Peclet numbers $P e_{c}$ and $P e_{T}$.}

Figure 8 illustrates the particular effects of two dimensionless Peclet numbers $P e_{c}$ (illustrating axial dispersion of concentration) and $P e_{T}$ (describing axial thermal heat conductivity) (c.f. Eq. (15)) on the mass and temperature profiles for the ratio $C_{S} / C_{L}=1$ and keeping $T_{\mathrm{init}}=T_{\mathrm{inj}}=T_{\text {ref }}$.

Figure 8(a) displays the effect of $P e_{c}$ for a fixed value of $P e_{T}=1200$. A change in $P e_{c}$ significantly influence the concentration and temperature peaks. The broadness of concentration and temperature profiles increases on decreasing the value of $P e_{c}$ (i.e. with increasing $\left.D_{L}\right)$.

In addition, Figure $8(\mathrm{~b})$ describes the influence of $P e_{T}$ (which contains the axial thermal heat conductivity coefficient $\lambda_{L}$, see Eq. (15)) on the concentration and temperature profiles for a fixed $P e_{c}=600$. It can be seen that for the parameters considered $P e_{T}$ effects only the peaks of temperature, while it does not influence the peak of concentration.

Figures 9 and 10 demonstrate the effects of $P e_{c}$ and $P e_{T}$ on the first two moments (Eqs. (S2-5) and (S2-8)-(S2-11)) representing retention time and variance of the profiles. In the calculations performed, we have chosen the orders of magnitude as $C_{S}=4 \mathrm{~J} / \mathrm{cm}^{3} \mathrm{~K}$ and $C_{L}=4 \mathrm{~J} / \mathrm{cm}^{3} \mathrm{~K}$. It is evident that Peclect numbers $P e_{c}$ and $P e_{T}$ do not influence the 
mean retention times $\left(\mu_{11}\right.$ and $\left.\mu_{22}\right)$. On the other hand, the variances $\left(\sigma_{11}\right.$ and $\left.\sigma_{22}\right)$ can decrease, stay constant or increase as functions of the values of $P e_{c}$ and $P e_{T}$.

\section{Influence of $u$ on HETP curves}

Second moments are particularly interesting for separation in chromatography, typically regarded as tools for assessing efficiency of the column. These moments are often expressed as "heights equivalent to theoretical plates (HETP)". ${ }^{5}$

Figure 11 shows for illustration plots of HETP curves as functions of the linear velocity for the reference parameters and different values of the mass and heat transfer coefficients $k$ and $h$. The two types of HETP values were calculated according to the following classical equations: ${ }^{5,46}$

$$
\operatorname{HETP}_{c}=\frac{L \sigma_{11}}{\left(\mu_{11}^{1}\right)^{2}}, \quad \operatorname{HETP}_{T}=\frac{L \sigma_{22}}{\left(\mu_{22}^{1}\right)^{2}}
$$

The $\operatorname{HETP}_{c}$ value corresponds to the coupled concentration and temperature peaks, while the $\mathrm{HETP}_{T}$ value corresponds to the decoupled temperature peak.

In the calculated results of Figure 11, we took the reference values of parameters listed in Table 1. It can be observed in Figure 11 that, depending on $k$ and $h$, either the coupled peaks of concentration and temperature or the decoupled peak of temperature can be more dispersed.

\section{Conclusion}

The governing equations of a linearized adiabatic non-equilibrium lumped kinetic model (LKM) were solved analytically in order to evaluate the influence of inherent temperature fluctuations on the liquid chromatographic processes. The model incorporated both mass 
and heat transfer resistances in the solid phase. The Laplace transformation and linear transformation techniques were used as basic tools to decouple the governing set of coupled differential equations. The solutions obtained could be very helpful to understand, analyze and interpret the concentration and temperature profiles in chromatographic columns when variations from isothermal condition are relatively small. For further detailed analysis, instructive analytical expressions of the first two temporal moments were derived. In order to identify the model parameters from experimentally obtained elution profiles condensed into the moments and to understand the traveling phenomenon completely, the derived moment expressions are of great significance. Their exploitation requires accurate measurements of the profiles allowing to determine reliably higher order moments. This might be experimentally difficult and requires precise detection. Several case studies were provided involving the application of estimated reasonable model parameters. To verify the obtained analytical solutions and to determine their ranges of applicability, the analytical results for linearized isotherms were compared with the numerical results of a high resolution finite volume scheme incorporating nonlinear isotherms. The derived solutions of this paper can be utilized in further systematic studies to find a general criteria that could allow predicting maximum variations from the isothermal condition and offering a rational basis for deciding under which conditions a non-isothermal model should be used.

\section{Supporting Information}

The Supporting Information is available free of charge on the ACS Publications website. Appendix S1: Analytical solutions.

Appendix S2: Analytical moments.

Acknowledgement: We are thankful to Higher Education Commission (HEC) of Pakistan for financial support under Project nr. 1550. 


\section{References}

(1) Fanali, S.; Haddad, P. R.; Poole, C.; Riekkola, M. -L. Liquid Chromatography: Fundamentals and Instrumentation; 2nd ed.; Elsevier: The Netherlands, 2017.

(2) Snyder, L. R.; Kirkland, J. J.; Dolan, J. W. Introduction to Modern Liquid Chromatography; 3rd ed.; John Wiley \& Sons, Inc, Hoboken: New Jersey, 2010.

(3) Guiochon, G. Preparative Liquid Chromatography. J. Chromatogr. A 2002, 965, 129.

(4) Guiochon, G.; Lin, B. Modeling for Preparative Chromatography; Elsevier Academic Press: New York, 2003.

(5) Guiochon, G.; Felinger, A.; Shirazi, D. G.; Katti, A. M. Fundamentals of Preparative and Nonlinear Chromatography; 2nd ed.; ELsevier Academic Press; New York, 2006.

(6) Cerro, R. L.; Smith, w. Effects of Heat Release and Nonlinear Equilibrium on Transient Adsorption. Ind. Eng. Chem. Fund. 1969, 8, 796.

(7) Haynes Jr., H. W. An Analysis of Sorption Heat Effects in the Pulse Gas Chromatography Diffusion Experiment. AIChE J. 1986, 32, 1750.

(8) Zhong, G. M.; Meunier, F. Interference and Heat Effects: Moment Analysis for Twocomponent Chromatography. J. Chromatogr. A 1994, 658, 355.

(9) Kruglov, A. Methanol Synthesis in a Simulated Countercurrent Moving-bed Adsorptive Catalytic Reactor. Chem. Eng. Sci. 1994, 49, 4699.

(10) Yongsunthon, I.; Alpay, E. Design of Periodic Adsorptive Reactors for the Optimal Integration of Reaction, Separation and Heat Exchange. Chem. Eng. Sci. 1999, 54, 2647. 
(11) Xiu, G.; Li, P.; Rodrigues, A. E. Sorption-enhanced Reaction Process with Reactive Regeneration. Chem. Eng. Sci. 2002, 57, 3893.

(12) Glöckler, B.; Dieter, H.; Eigenberger, G.; Nieken, U. Efficient Reheating of a Reverseflow Reformer-An experimental study. Chem. Eng. Sci. 2007, 62, 5638.

(13) Brandt, A.; Mann, G.; Arlt, W. Temperature Gradients in Preparative Highperformance Liquid Chromatography Columns. Chromatogr. A 1997, 769, 109.

(14) McCalley, D. V. Effect of Temperature and Flow-rate on Analysis of Basic Compounds in High-performance Liquid Chromatography Using a Reversed-phase Column. J. Chromatogr A. 2000, 902, 311.

(15) Sainio, T. Ion-exchange Resins as Stationary Phase in Reactive Chromatography. Ph.D. Dissertation, Lappeenranta University of Technology, Finland, 2005.

(16) Sainio, T.; Kaspereit, M.; Kienle, A.; Seidel-Morgenstern, A. Thermal Effects in Reactive Liquid Chromatography. Chem. Eng. Sci. 2007, 62, 5674.

(17) Gu, B.; Corte, H.; Luong, J.; Pursch, M.; Eckerle, P.; Mustacich, R. Low Thermal Mass Liquid Chromatography. Anal. Chem. 2009, 81, 1488.

(18) Sainio, T.; Zhang, L.; Seidel-Morgenstern, A. Adiabatic Operation of Chromatographic Fixed-bed Reactors. Chem. Eng. J. 2011, 168, 861.

(19) Vu, T. D.; Seidel-Morgenstern, A. Quantifying Temperature and Flow Rate Effects on the Performance of a Fixed-bed Chromatographic Reactor. J. Chromatogr. A 2011, $1218,8097$.

(20) Javeed, S.; Qamar, S.; Seidel-Morgenstern, A.; Warnecke, G. Parametric Study of Thermal Effects in Reactive Liquid Chromatography. Chem. Eng. J. 2012, 191, 426. 
(21) Graça, N. S.; Pais, L. S.; Silva, V. M. T. M.; Rodrigues, A. E. Thermal Effects on the Synthesis of 1,1-dibutoxyethane in a Fixed-bed Adsorptive Reactor. Chem. Eng. Tech. 2012, 35, 1989.

(22) Graça, N. S.; Pais, L. S.; Silva, V. M. T. M.; Rodrigues, A. E. Thermal Effects on the Synthesis of Acetals in a Simulated Moving Bed Adsorptive Reactor. Chem. Eng. J. 2012, 207-208, 504.

(23) Qamar, S.; Sattar, F. A.; Seidel-Morgenstern, A. Theoretical Investigation of Thermal Effects in Non-isothermal Non-equilibrium Reactive Liquid Chromatography. Chem. Eng. Res. Design 2016, 115, 145.

(24) Qamar, S.; Sattar, F. A.; Batool, I.; Seidel-Morgenstern, A. Theoretical Analysis of the Influence of Forced and Inherent Temperature Fluctuations in an Adiabatic Chromatographic Column. Chem. Eng. Sci. 2017, 161, 249.

(25) Van Genuchten, M. Th. Analytical Solutions for Chemical Transport with Simultaneous Adsorption, Zero-order Production and First-order Decay. J. Hydrology 1981, $49,213$.

(26) Rice, R. G.; Do, D. D. Applied Mathematics and Modeling for Chemical Engineers; Wiley-Interscience: New York, 1995.

(27) Javeed, S.; Qamar, S.; Ashraf, W.; Warnecke, G.; Seidel-Morgenstern, A. Analysis and Numerical Investigation of Two Dynamic Models for Liquid Chromatography. Chem. Eng. Sci. 2013, 90, 17.

(28) Durbin, F. Numerical Inversion of Laplace Transforms: An Efficient Improvement to Dubner and Abate's Method. Comput. J. 1974, 17, 371. 
(29) Kubin, M. Beitrag zur Theorie der Chromatographie. Collect. Czech. Chem. Commun. 1965, 30, 1104.

(30) Kubin, M. Beitrag zur Theorie der Chromatographie. 11. Einfluss der Diffusion Ausserhalb und der Adsorption Innerhalb des Sorbens-Korns. Collect. Czech. Chem. Commun. 1965, 30, 2900.

(31) Kucera, E. Contribution to the Theory of Chromatography: Linear Non-equilibrium Elution Chromatography. J. Chromatogr. A 1965, 19, 237.

(32) Schneider, P.; Smith, J. M. Adsorption Rate Constants from Chromatography. AIChE J. 1968, 14, 762 .

(33) Suzuki, M. Notes on Determining the Moments of the Impulse Response of the Basic Transformed Equations. J. Chem. Eng. Japan 1973, 6, 540.

(34) Wolff, H. -J.; Radeke, K. -H; Gelbin, D. Heat and Mass Transfer in Packed Beds-IV: Use of Reighted Moments to Determine Axial Dispersion Coefficient. Chem. Eng. Sci. 1980, 34, 101.

(35) Wolff, H. -J.; Radeke, K. -H; Gelbin, D. Weighted Moments and the Pore-diffusion Model. Chem. Eng. Sci. 1980, 35, 1481.

(36) Lenhoff, A. M. Significance and Estimation of Chromatographic Parameters. J. Chromatogr. A 1987, 384, 285.

(37) Miyabe, K.; Guiochon, G. Influence of the Modification Conditions of Alkyl Bonded Ligands on the Characteristics of Reversed-phase Liquid Chromatography. J. Chromatogr. A 2000, 903, 1.

(38) Miyabe, K.; Guiochon, G. Measurement of the Parameters of the Mass Transfer Kinetics in High Performance Liquid Chromatography. J. Sep. Sci. 2003, 26, 155. 
(39) Miyabe, K. Surface Diffusion in Reversed-phase Liquid Chromatography Using Silica Gel Stationary Phases of Different C1 and C18 Ligand Densities. J. Chromatogr. A 2007, 1167, 161.

(40) Miyabe, K. Moment Analysis of Chromatographic Behavior in Reversed-phase Liquid Chromatography. J. Sep. Sci. 2009, 32, 757.

(41) Qamar, S.; Seidel-Morgenstern, A. Extending the Potential of Moment Analysis in Chromatography. Tends Analyt. Chem. 2016, 81, 87.

(42) Javeed, S.; Qamar, S.; Seidel-Morgenstern, A.; Warnecke, G. Efficient and Accurate Numerical Simulation of Nonlinear Chromatographic Processes. Comput. 83 Chem. Eng. 2011, 35, 2294.

(43) Gritti, F.; Gilar, M.; Jarrell, J. A. Quasi-adiabatic Vacuum-based Column Housing for Very High-pressure Liquid Chromatography. J. Chromatogr. A 2016, 1456, 226.

(44) Sajonz, P.; Guan-Sajonz, H.; Zhong, G.; Guiochon, G. Application of the Shock Layer Theory to the Determination of the Mass Transfer Rate Coefficient and its Concentration Dependence for Proteins on Anion Exchange Columns. Biotechnol. Prog. 1997, 12, 170.

(45) Kaczmarski, K.; Antos, D.; Sajonz, H.; Sajonz, P.; Guiochon, G. Comparative Modeling of Breakthrough Curves of Bovine Serum Albumin in Anion-exchange Chromatography. J. Chromatogr. A 2001, 925, 1.

(46) Van Deemter, J. J.; Zuiderweg, F. J.; Klinkenberg, A. Longitudinal Diffusion and Resistance to Mass Transfer as Causes of Nonideality in Chromatography. Chem. Eng. Sci. 1956, 5, 271. 
Table 1: Reference parameters used in case studies.

\begin{tabular}{|l|l|}
\hline Parameters & Values \\
\hline \hline Length of column & $L=10 \mathrm{~cm}$ \\
Porosity & $\epsilon=0.4$ \\
Radius of particle & $R_{p}=0.004 \mathrm{~cm}$ \\
Interstitial velocity & $u=2.4 \mathrm{~cm} / \mathrm{min}$ \\
Henry's constant & $a_{\text {ref }}=1.2$ \\
Coefficient of dispersion & $D_{L}=0.04 \mathrm{~cm}^{2} / \mathrm{min}$ \\
Coefficient of heat conduction & $\lambda_{L}=0.08 \mathrm{~J} / \mathrm{cm} \mathrm{min}$ \\
Enthalpy of adsorption & $\Delta H_{A}=-2 \mathrm{~kJ} / \mathrm{mol}$ \\
Mass transfer coefficient & $k=1 \mathrm{~min}^{-1}$ \\
Heat transfer coefficient & $h=0.1 \mathrm{~J} / \mathrm{min} \mathrm{cm}^{2} \mathrm{~K}$ \\
Density times heat capacity of mobile phase & $C_{L}=4 \mathrm{~J} / \mathrm{cm}^{3} \mathrm{~K}$ \\
Density times heat capacity of solid phase & $C_{S}=4 \mathrm{~J} / \mathrm{cm}^{3} \mathrm{~K}$ \\
Initial concentration & $c_{\text {init }}=0 \mathrm{~mol} / l$ \\
Initial temperature & $T_{\text {init }}=300 \mathrm{~K}$ \\
Injected concentration & $c_{\text {inj }}=1 \mathrm{~mol} / l$ \\
Injected temperature & $T_{\text {inj }}=300 \mathrm{~K}$ \\
Reference temperature & $T_{\text {ref }}=300 \mathrm{~K}$ \\
Time of injection & $t_{\text {inj }}=5 \mathrm{~min}$ \\
\hline
\end{tabular}



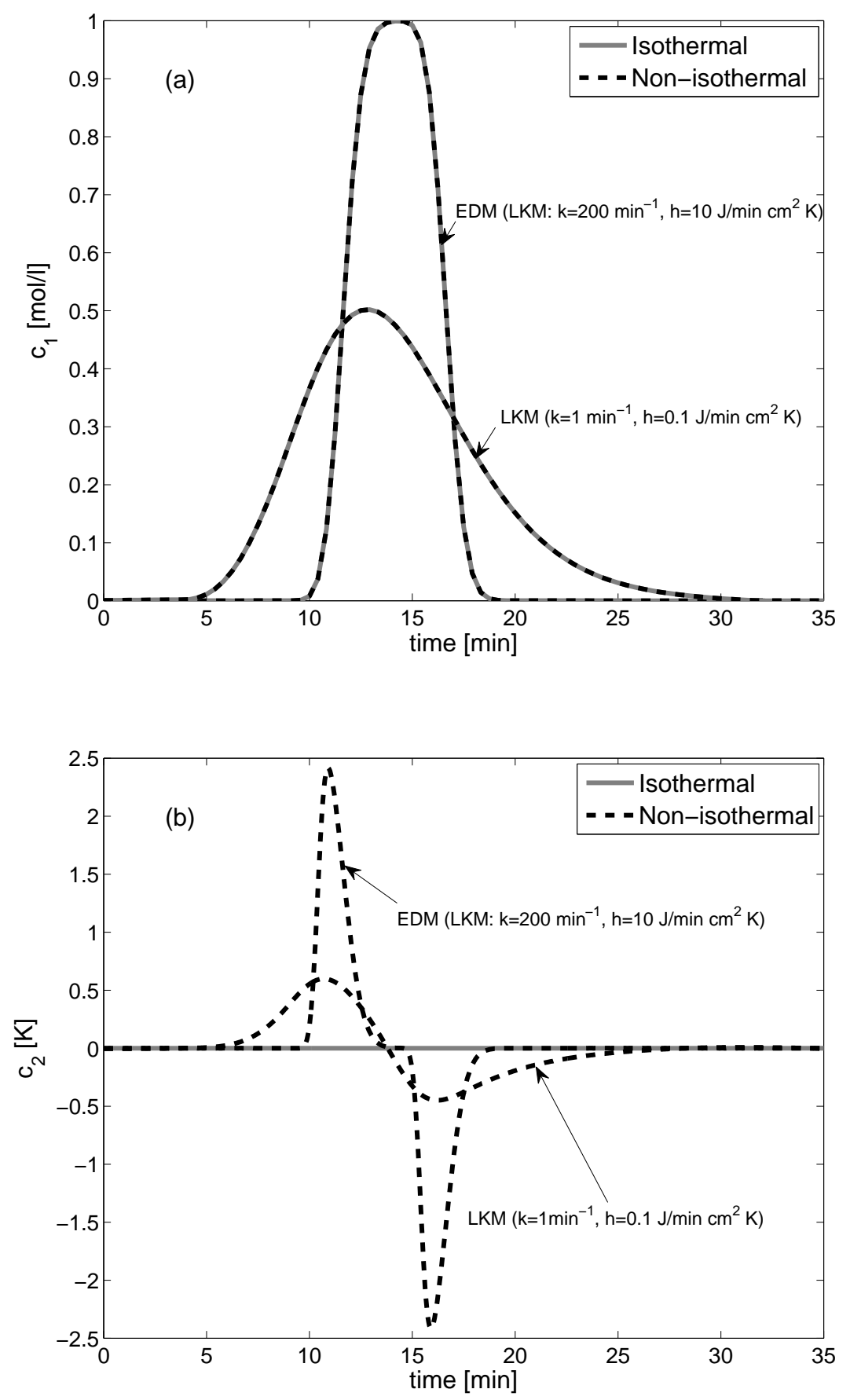

Figure 1: Comparison of LKM and EDM solutions for isothermal $\left(\Delta H_{A}=0\right)$ and nonisothermal $\left(\Delta H_{A}=-2 \mathrm{~kJ} / \mathrm{mol}\right)$ cases considering the reference parameters given in Table 1. Here, $c_{1}=c$ and $c_{2}=T-T_{\text {ref }}$ denote the liquid phase concentration and temperature, respectively. 

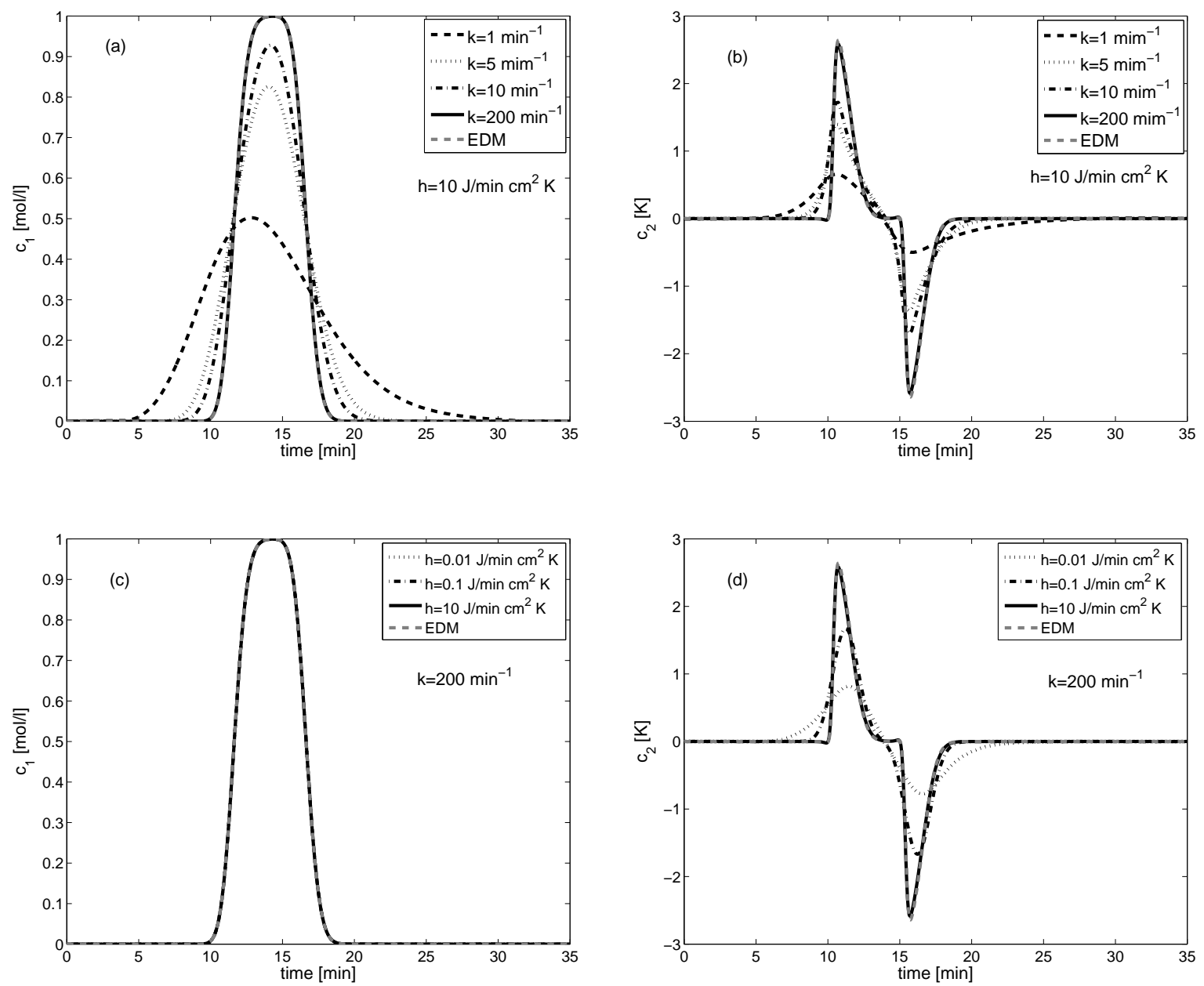

Figure 2: Effects of mass transfer rate coefficient $k$ (plots (a) and (b)) and heat transfer coefficient $h$ (plots $(\mathrm{c})$ and $(\mathrm{d}))$ on concentration $\left(c_{1}\right)$ and temperature $\left(c_{2}=T-T_{\text {ref }}\right)$ profiles for the reference parameters given in Table 1 . Here, $\Delta H_{A}=-2 \mathrm{~kJ} / \mathrm{mol}$ and $k$ and $h$ as indicated. 

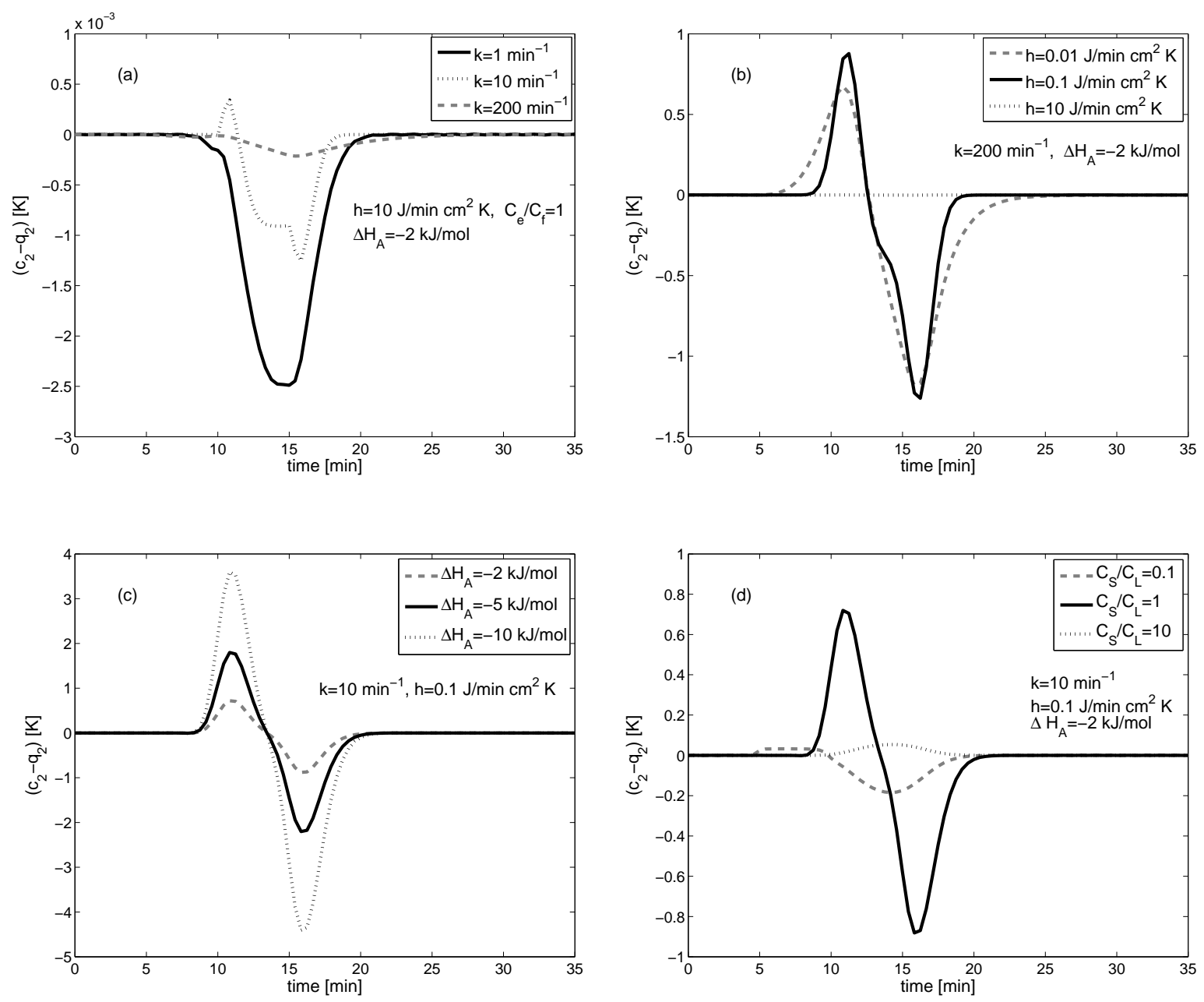

Figure 3: Effects of several parameters on the differences between liquid and solid temperatures (i.e. $\left(c_{2}-q_{2}\right)$ or $\left.\left(T-T_{S}\right)\right)$ for the reference parameters except the parameters indicated. 

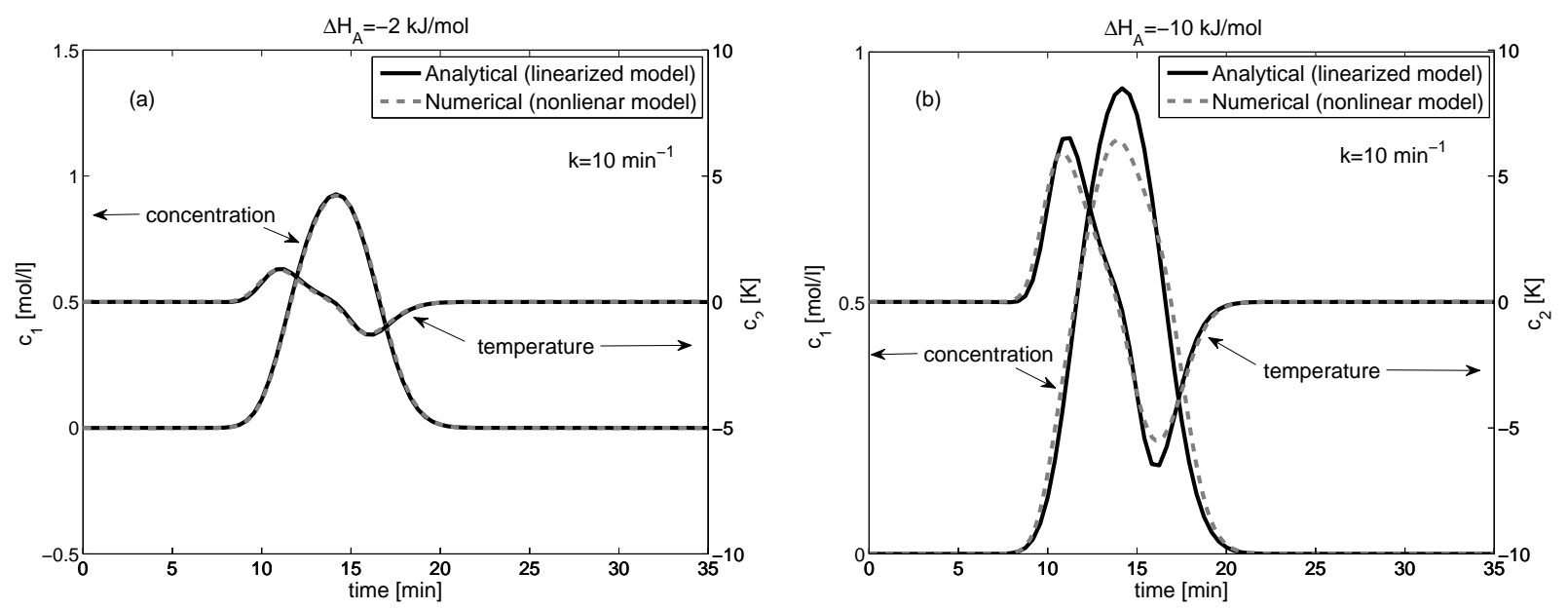

Figure 4: A comparison of concentration profiles obtained through analytical solutions (for linearized model based on Eq. (22)) and through numerical solutions (for nonlinear model based on Eq. (6)) assuming different values of the enthalpy of adsorption $\Delta H_{A}$. The parameters correspond to Table 1 , except $k=10 \mathrm{~min}^{-1}$.
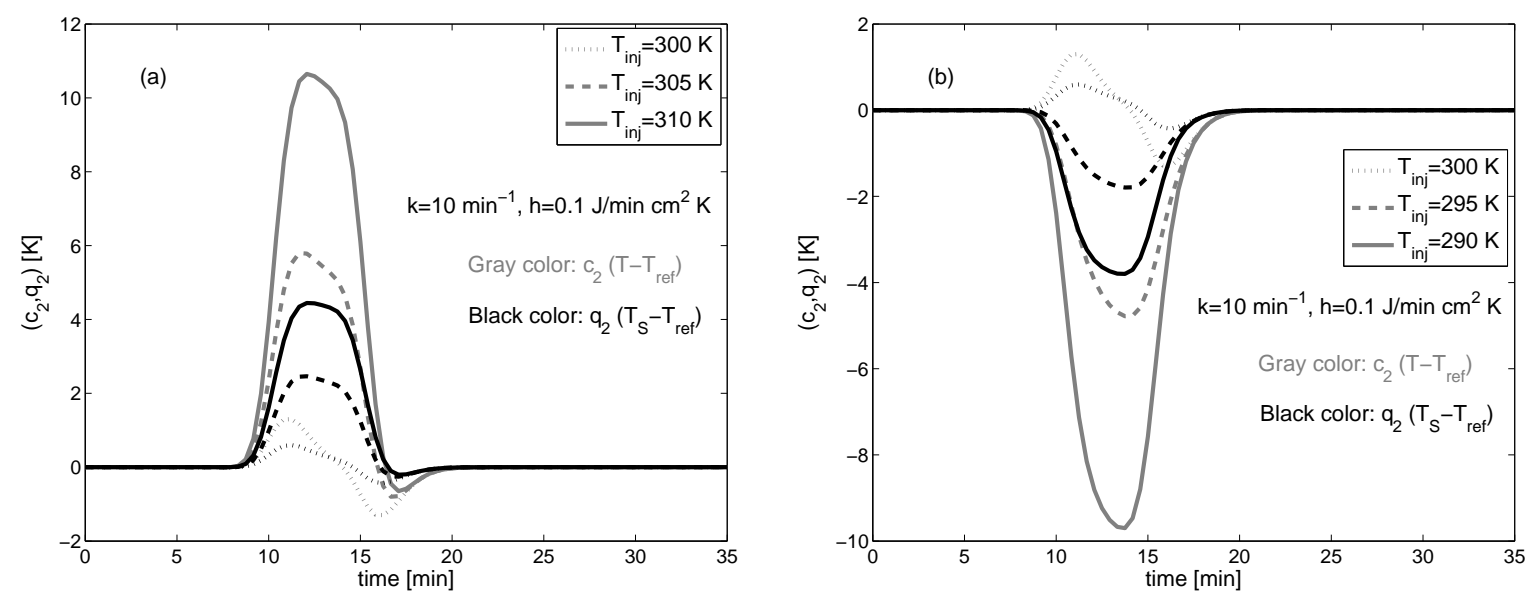

Figure 5: Effect of $T_{\text {inj }} \neq T_{\text {ref }}$ on concentration and temperature profiles for $\Delta H_{A}=$ $-2 \mathrm{~kJ} / \mathrm{mol}$. The parameters correspond to Table 1 , except $k=10 \mathrm{~min}^{-1}$. 

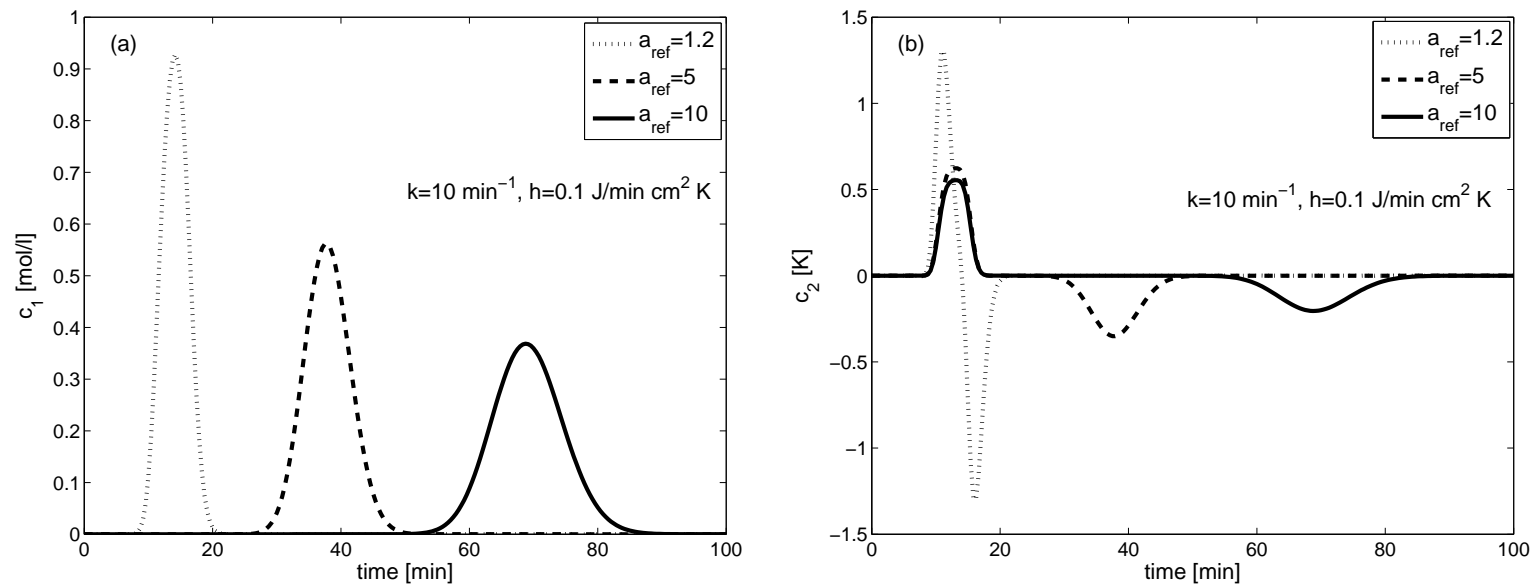

Figure 6: Effect of Henry's constant $a_{\text {ref }}$ on the concentration and temperature profiles for the reference parameters and $\Delta H_{A}=-2 \mathrm{~kJ} / \mathrm{mol}$. 

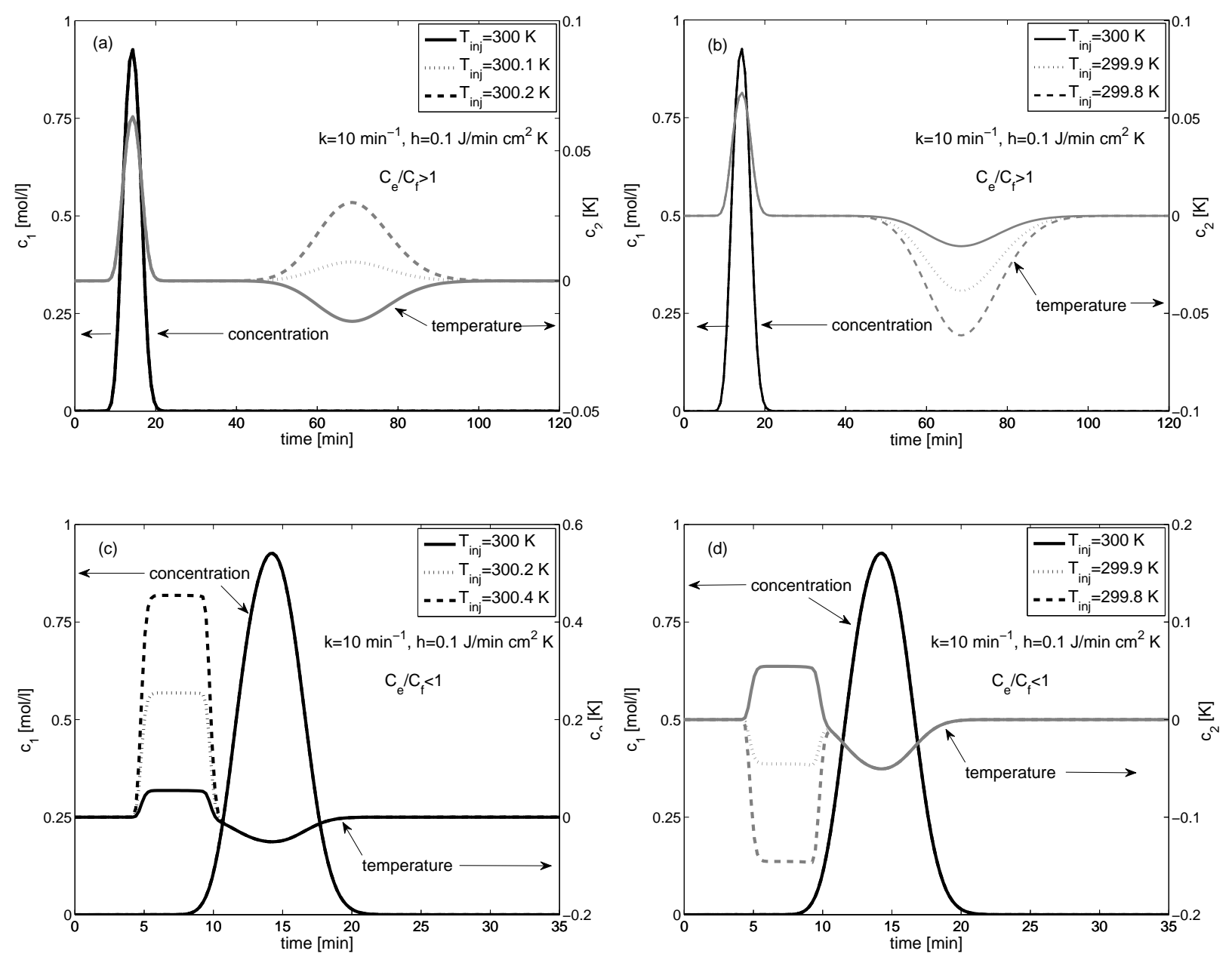

Figure 7: Effects of the ratio $C_{S} / C_{L}$ and $T_{\text {inj }}$ on the concentration and temperature profiles for $\Delta H_{A}=-2 \mathrm{~kJ} / \mathrm{mol}$. For plots (a) \& (b): $C_{S}=40 \mathrm{~J} / \mathrm{cm}^{3} \mathrm{~K}$ and $C_{L}=4 \mathrm{~J} / \mathrm{cm}^{3} \mathrm{~K}$, while for plot (c) \& (d): $C_{S}=4 \mathrm{~J} / \mathrm{cm}^{3} \mathrm{~K}$ and $C_{L}=40 \mathrm{~J} / \mathrm{cm}^{3} \mathrm{~K}$. 

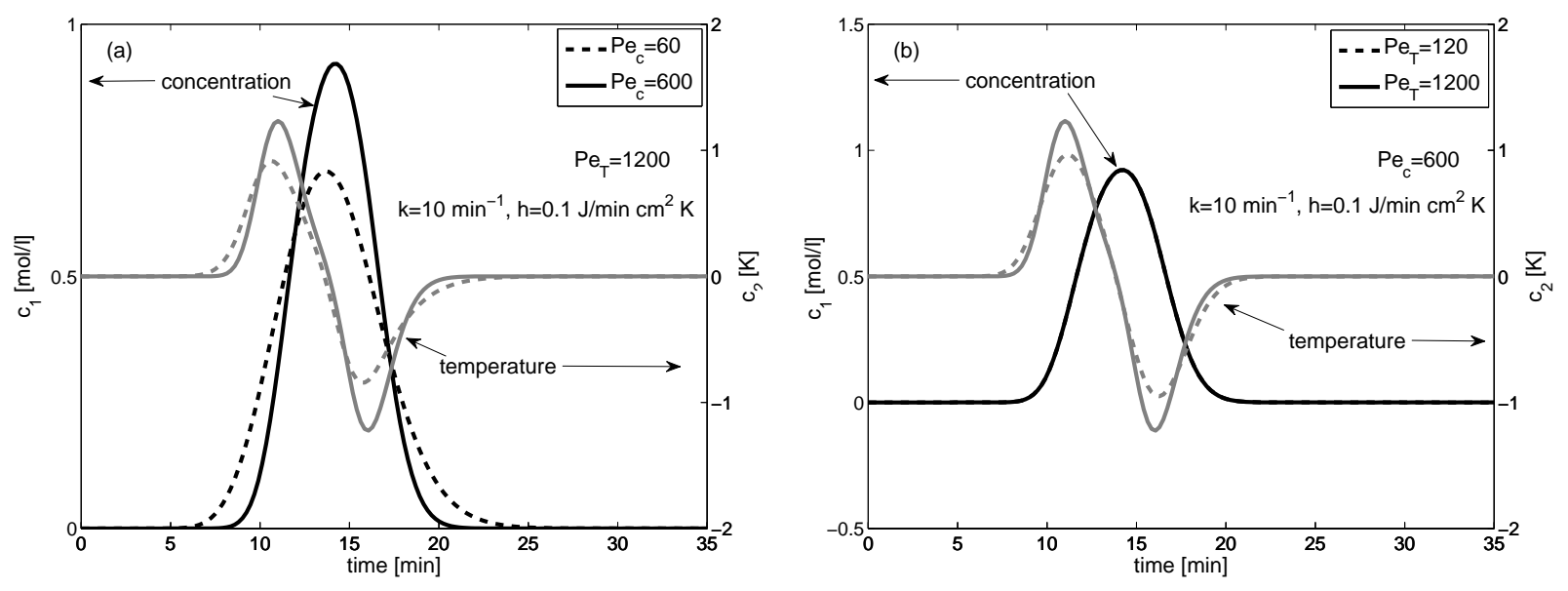

Figure 8: Effects of Peclet numbers $P e_{c}$ and $P e_{T}$ on the concentration and temperature profiles. Here, $C_{S}=40 \mathrm{~J} / \mathrm{cm}^{3} K$ and $C_{L}=4 \mathrm{~J} / \mathrm{cm}^{3} K$ for plots (a) and (b), while $C_{S}=$ $4 \mathrm{~J} / \mathrm{cm}^{3} \mathrm{~K}$ and $C_{L}=40 \mathrm{~J} / \mathrm{cm}^{3} \mathrm{~K}$ for plots (c) and (d). Moreover, the reference parameters in Table 1 hold together with $\Delta H_{A}=-10 \mathrm{~kJ} / \mathrm{mol}$ and $k=$ and $h$ as indicated.
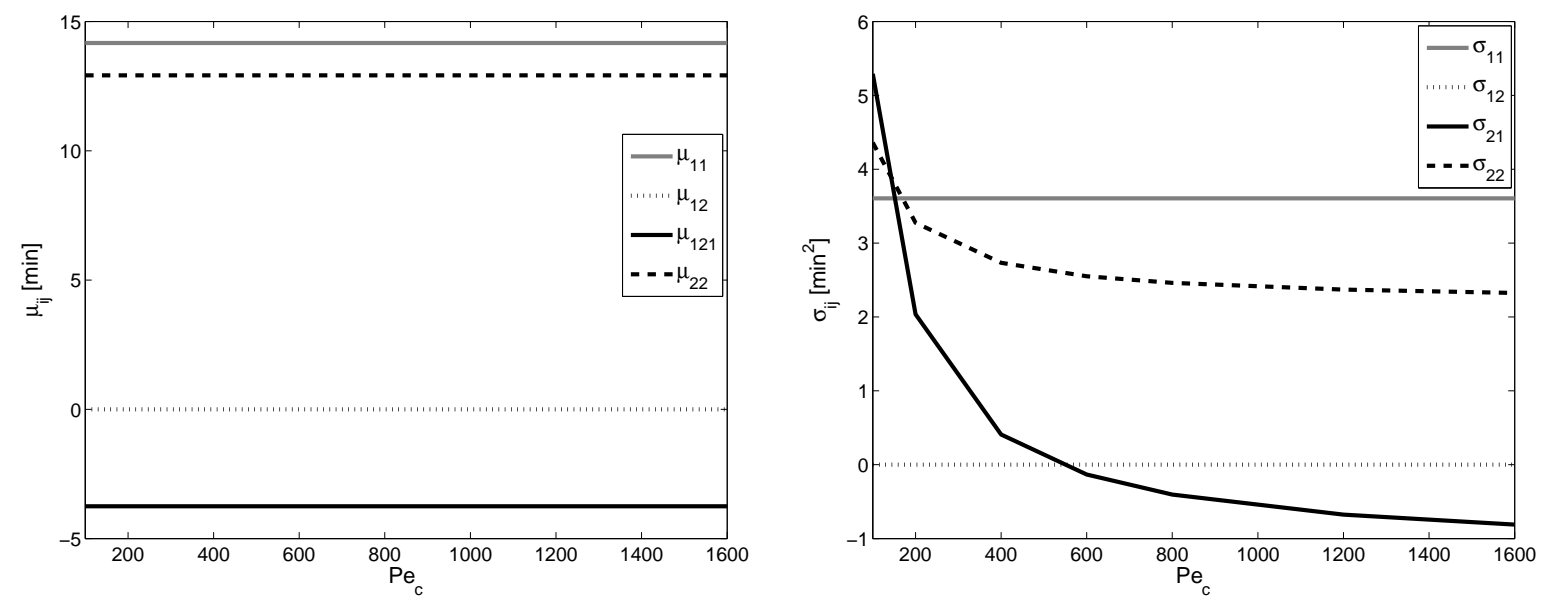

Figure 9: Effect of $P e_{c}$ on concentration and temperature moments for a fixed $P e_{T}=$ 1200. Here, $k=10 \mathrm{~min}^{-1}, h=0.1 \mathrm{~J} / \mathrm{min} \mathrm{cm}^{2} K, \Delta H_{A}=-2 \mathrm{~kJ} / \mathrm{mol}$ and the reference parameters. 

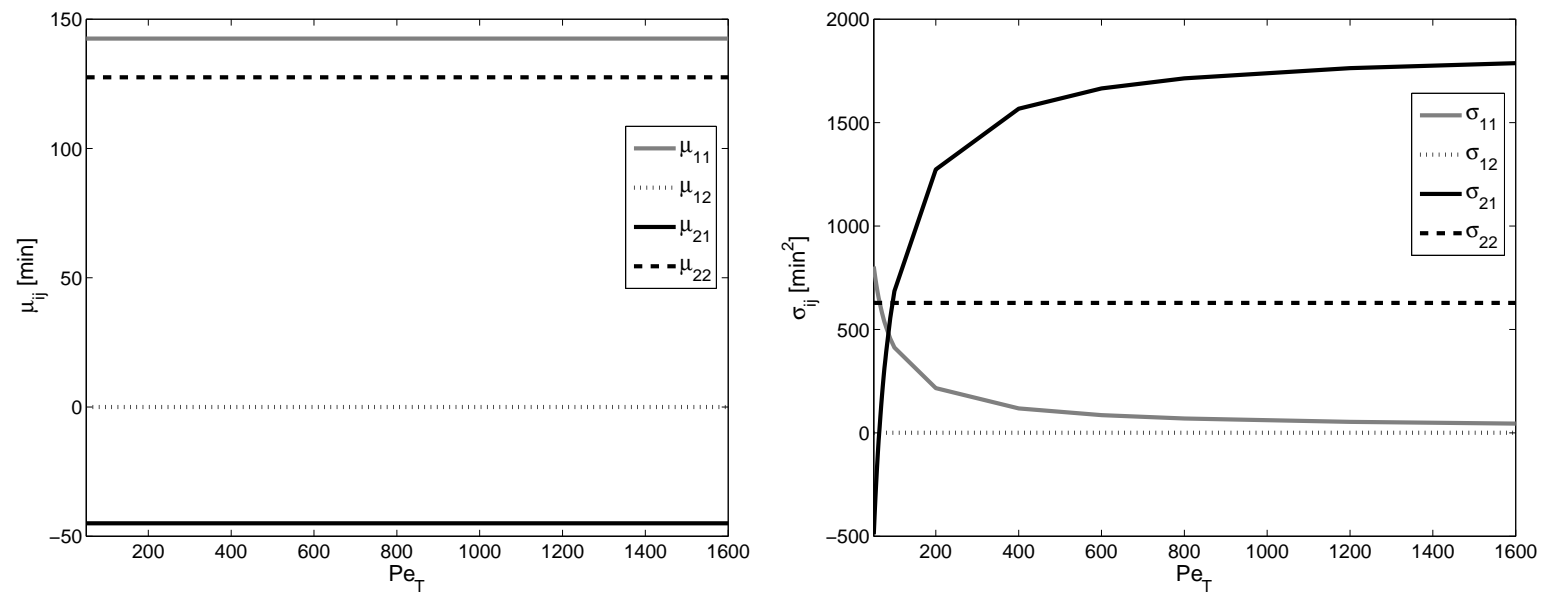

Figure 10: Effect of $P e_{T}$ on concentration and temperature moments for a fixed $P e_{c}=$ 600. Here, $k=10 \mathrm{~min}^{-1}, h=0.1 \mathrm{~J} / \mathrm{min} \mathrm{cm}^{2} \mathrm{~K}, \Delta H_{A}=-2 \mathrm{~kJ} / \mathrm{mol}$ and the reference parameters. 

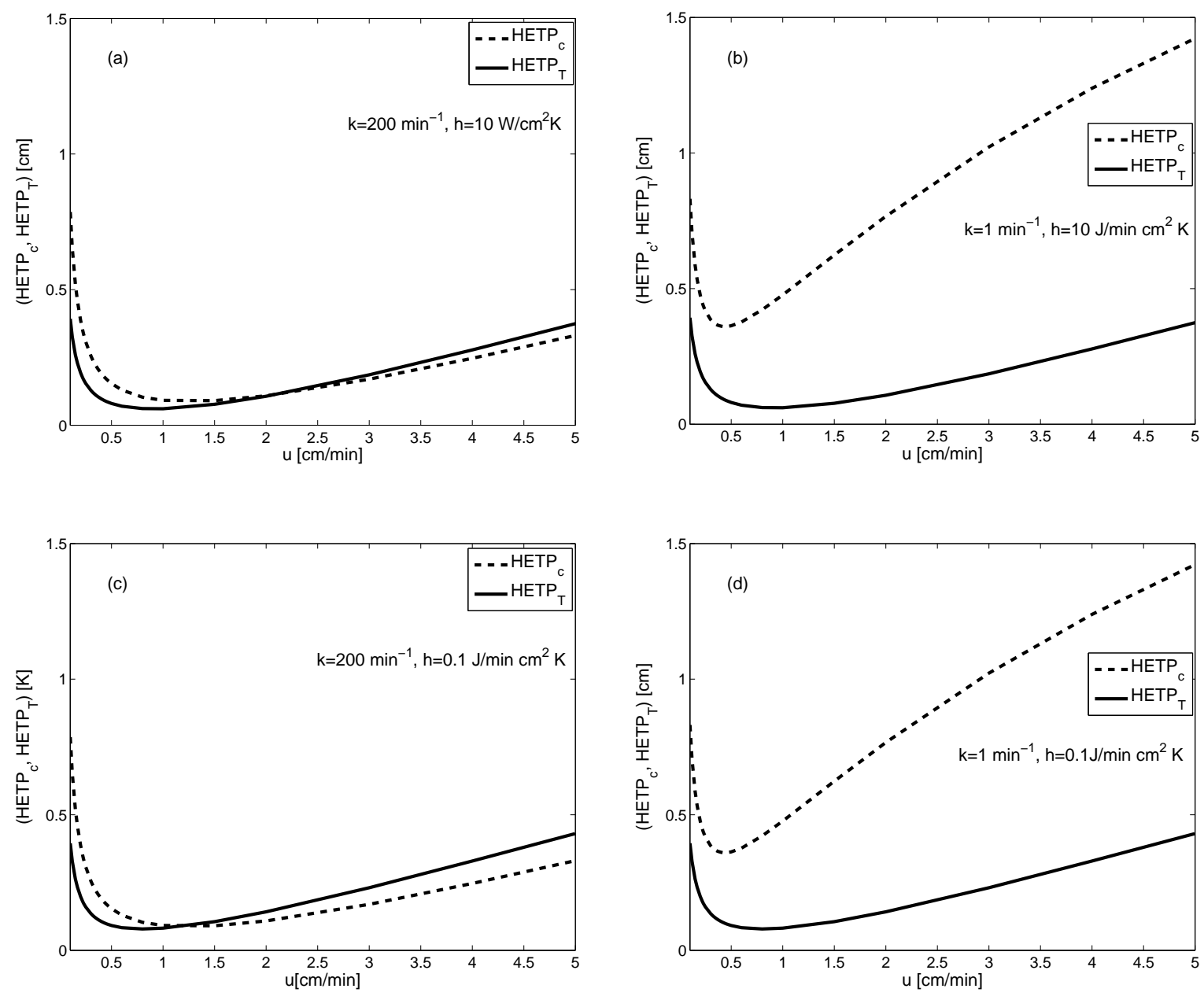

Figure 11: Plot (a): Plots of two types of HETP curves (c.f. Eq. (27)) as functions of $u$ for different values of $k$ and $h$. Here, $\Delta H_{A}=-2 k J / m o l$ together with reference parameters given in Table 1 and values of $k$ and $h$ as indicated. 


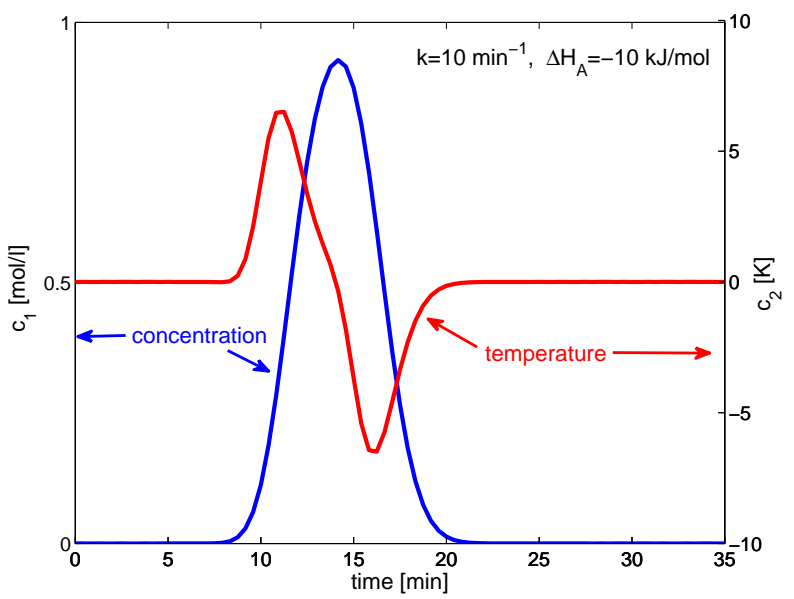

For Table of Contents only. 